\title{
L'elettronica nella tecnica aerospaziale $\left(^{*}\right)$
}

\author{
F. SALZA
}

\author{
Ricevuto il 19 Febbraio 1963
}

Riassunto. - L'elettronica reca un contributo essenziale alla tecnica aerospaziale in ogni fase di qualsiasi sua realizzazione.

In fase di progetto è fondamentale l'impiego del calcolo analogico o digitale, di quello analogico-digitale e delle tecniche di simulazione.

Nel volo spaziale la guida e la stabilizzazione si basano sull uso di sistemi elettronici che sono anche impiegati largamente nella essenziale fase di preparazione al volo.

Così pure le comunicazioni coi passeggeri e le telemisure sono possibili soltanto grazie all'impiego delle tecniche più avanzate delle telecommicazioni.

Si esamina infine la situazione attuale per ciò che si riferisce al grado di affidamento delle apparechiature elettroniche in conseguenza della loro crescente complessità: l'impiego di elementi allo stato solido e di adeguate tecniche circuitali consente di conservarlo ad un valore accettabile nonostante la crescente complicazione degli apparati in conseguenza delle sempre più gravi esigenze.

SUMMAr. - The role of Electronics in each phase of all space projects is essential.

In the design phase analogue, digital, digital-analogue electronic computation and simulation techniques are heavily relied upon.

During space flight guidance and stabilization are achieved with electronies and likewise electronics are widely used in all pre-flight phases.

Space communication in manned and unmanned flight and telemetering must use the most sophisticated communications techniques.

Electronic equipment is becoming increasingly complicated: its reliability is briefly discussed. Solid state electronics and advanced circuit design seem to give satisfactory reliability even with the increased demands placed upon electronic equipment.

$\left(^{*}\right)$ Nota presentata al $2^{\circ}$ Congresso Internazionale Tecnico Scientifico dello Spazio. Roma, 19-23 Giugno 1962. 
Lo sviluppo della teconica aerospaziale is strettamente legato ad un largo impiego della tecnica elettronica. Credo che questa aflermazione di carattere generale possa essere accettata senz'altro: le realizzazioni già attuate e, certamente, anche quelle a cui oggi si tende, non solo non avrebbero potuto essere concepite e raggiunte, ma nemmeno essere verificate, senza l'ausilio della tecnical elettronica e, in particolare, della applicazione a cui si pensa immediatamente ogni volta che si nomina l'elettronica: la tecnica delle radiocommicazioni, che ha ormai raggiunto attraverso alla teoria dell'informazione un inquadramento preciso per (eio che si riferisee alla definizione della capacità limite di trasmissione.

Per illustrare il contributo dell'elettronica alla teconica acespaziale pensiamo di seguire la grenesi di un reicolo spaziale, dalla fase di concezione attraverso l'intero sviluppo e fino all'impiego, softermandoci in (ciascuna fase per esaminare le possibilità di utilizzazione e le contribuzioni eflettive della tecnica elettronica. Vedremo così che le une e le altre sono assai numerose e che spesso l'elettronica fornisce l'unica soluzione possibile a molti dei problemi che si presentano nel corso del progetto e della realizzazione.

Cominciamo dalla fase iniziale di concezione; la definizione dell'obbiettivo da raggiungere nasce certamente da una individuazione di possibilità al di là dello stato presente della tecnica e, se vogliamo veramente dare un nome alla causa prima dell'impostazione di ogni nuovo progetto, non puo essercene che uno: lo sforzo creativo ed immaginativo che è all'origine di ogni nostro progresso, di ogni nostra conquista.

Na, immediatamente, l'idea iniziale deve essere sottoposta all'indagine seientifica e si può dire chè a cominciare dalla fase di analisi preliminare sulla possibilità di attuazione (feasibility) fino alla definizione funzionale completa, in ogni progetto aerospaziale il contributo dellelettronica, attraverso i procedimenti di calcoli e di simulazione, i estremamente esteso.

I calcolatori analogici a numerici rappresentano lo strumento di lavoro più importante e più largamente usato in questo settore: converrà quindi sofiermarsi brevemente sulle possibilità e sugli orientamenti orlierni.

Sia il calcolo amalogo che quello numerico, come is noto, devono le loro odierne estese possibilità all'impiego dell'elettronica.

Fino a poco tempo fa si è parlato, in generale, di campi diversi di applicazione per i calcolatori dei due tipi.

Per i calcolatori analogici: calcolo con approssimazioni ridotte, ma con facile variabilità dei parametri di base del sistema. Contemporanea- 
mente difficoltà di impostazione per problemi con roefficienti variabili e nella realizzazione di operatori non lineari; infine una certa possibilità di soluzione in tempo reale, genemalmente tanto più limitata quanto più nel problema compaiono, appunto, operatori non lineari.

Per i calcolatori numerici, invece, si parla generalmente della possibilità di preceisioni assai più elevate, ma con maggriori difficoltà di variazione dei parametri nei problemi di ottimizzazione; difficoltà ancolä magrgiora di operare in tempo reale.

L'evoluzione dei calcolatori dei due tipi ha portato oggri sempre più, nella tecnica aerospaziale, al loro impiego promiscuo in complessi analogico-numerici: questa soluzione è stata resa possibile soprattutto dai sistemi rapidi per effettuare nei due sensi la conversione analogiconumerica, dall'arcorciamento dei tempi di accesso delle memorie a di esecuzione delle operazioni nella parte numerica.

Certamente, tra i numerosi fattori che hanno condotto ai più recenti progressi nei campo dei calcolatori, il maggior contributo singolo è quello doruto all'impiego di circuiti elettronici allo stato solido, sia per la maggior facilita di ottenere tempi brevi di commutazione che per la possibilità di aumentare il numero di elementi di calcolo ed ausiliari senza raggiungere configurazioni proibitive sia come ingombro che come potenza dissipata. Infine l'elettronica dello stato solido ha permesso di raggiungere gradi di afficlamento aceettabili anche in apparecchiature con numero assai elevato di componenti, riducendo dlasticamente i tempi di indisponibilità dei calcolatori.

Questo breve esame dell'uso del calcolo elettronico nella fase di progetto, per accertare la realizzabilità del sistema, stabilirne la configurazione ex oftimizzarlo, non sarebbe completo senza un cenno alle possibilità offerte dalla teconican di simulazione, intesa come sostituzione parziale, nel modello del sistema impostato sul calcolatore (analogico, numerico o misto) di componenti effettivi, già disponibili, del sistema in esame, collegati al modello da opportuni organi di trasduzione.

L'aumento della relocità nel calcolo elettronico accresce la possibilità di impiego dei calcolatori in tempo reale e ciò risulta particolarmente interessante, nel settore acrospaziale, sia per consentire l'impiego della simulazione nella fase di progetto di cui ci stiamo occupando sia, come vedremo in seguito, per attuare la guida in fase effettiva di impiego.

Continuando il nostro esame converrà ora aceennale all'impiego vastissimo di strumentazioni elettroniche per la realizzazione pratioa o la messa a punto degli elementi di sistema. 
Ricordiamo che, accanto all'impiego vastissimo dell'elettronica nelle tecniche delle misure a della registrazione, compare oggi quello, più propriamente tecnologico, di macchine operatrici a controllo elettronico per l'esecuzione di processi di fabbricazione, per esempio per la realizzazione di parti con configurazioni complesse, esprimibili in forma analitical o almeno approssimabili con una espressione analitica.

Passando ora alla fase di impiego del sistema ritroviamo ancora molteplici utilizzazioni delle apparecchiature elettroniche.

Parlando dell'impiego dei veicoli aerospaziali non si puó, prima di tutto, non esaminare quel complesso di operazioni di verifiea che preredono l'impiego effettivo. Nolte delle apparecehiature del veicolo e degli eventuali elementi di appoggio a terra sono di tipo elettronico. Inoltre, non fosse che per il numero spesso estremamente elevato di funzioni da analizzare, è spesso necessario ricorrere a schemi logici di verifica, impostati su calcolatori elettronici, per effettuare con sicurezza e senza errori l'intera sequenzal delle operazioni di controllo cui è necessario sottoporre il sistema.

Esaminando, successivamente, l'impiego effettivo del veicolo aerospaziale, osserviamo che all'elettronica sono sempre afficlate le funzioni di stabilizzazione e di guirla.

Circa la stabilizzazione è ben noto che, soprattutto nelle fasi in cui si utilizza un controllo aerodinamico, è raramente possibile conferire a un veicolo di prestazioni elevate una configurazione aerodinamica che soddisfi tutte le esigenze di stabilità: in questo caso l'elettronica offere il suo ausilio efficace consentendo di modificare nel senso desiderato le proprietà aerodinamiche intrinseche del veicolo attraverso una appropriata azione delle superfici di controllo.

Problemi analoghi trovano la loro soluzione attraverso l'elettronica nel caso della stabilizzazione attorno al baricentro dei veicoli spaziali, sia in fase propulsa che in fase orbitale o di volo nello spazio libero.

La guida del veicolo (moto del suo baricentro) può, in generale, essere attuata con sistemi inerziali, a programma oppure a comando da terra.

In ogni caso l'intervento di apparecchiature elettroniche è essenziale. Senza l'elettronica non potrebbero realizzarsi piattaforme inerziali, senza di essa non si potrebbe misurare, nello spazio, la posizione del veicolo e valutare l'entità dei comandi da impartire per arere la traicttoria residemata.

A questo proposito a importante notare quanto sia utile potere effettuare, sul calcolatore di guida, i calcoli in tempo reale, sia per assi- 
curare la traiettoria prevista col minimo di provvedimenti correttivi, sia per accertare al più presto, in caso di inconvenienti di funzionamento, il manifestarsi di una situazione pericolosa.

E pressochè superfluo ricordare che nella funzione di guida si intende anche compreso, generalmente, tutto un complesso di operazioni ausiliarie: accensione dei vari stadi, loro distacco, etc. in funzione di tempi prestabiliti o del raggiungimento di determinate condizioni cinematiche e che, anche per queste operazioni, siano esse programmate a priori o comandate in funzione dello svolgimento del lancio, il contributo dell'elettronicà è essenziale.

Questa esposizione non si occupa del problema delle commicazioni con veicoli spaziali; tuttavia faremo qualche considerazione specifica sulle apparecehiature elettroniche usate per tale scopo.

Considerando sia le prestazioni proprie del veicolo che la sua eventuale utilizzazione a scopi di ricerche deve in ogni caso essere risolto un problema di telemisura. Eceo una tecnica la cui nascita e il cui sviluppo sono essenzialmente legati all'elettronica: sia dal punto di vista della quantità di informazione da trasmettere, sia per ciò che riguarda semplicemente l'esigenza di ottenere questo complesso di dati su un canale di trasmissione radio, ai si trova di fronte a problemi che sono nati, si può dire, con la tecnica aerospaziale e che, risolti per merito dell'elettronica, sulle tecnica aerospaziale hanno trovato le loro prime appliazioni, anche se l'efficienza delle realizzazioni ha poi suggerito l'estensione del loro impiego in altri campi.

I rati trasmessi possono essere semplici rilevamenti di posizione, oppure si può avere una trasmissione automatica di dati di ogni genere, in tempo reale o differito, per acquisizione di informazioni sul comportamento dei veicolo e sull'ambiente in cui esso si trova, oppure ancora la trasmissione è destinata alla utilizzazione specifica del veicolo (ap)poggio di telecommicazioni, assistenza alla navigazione) ed alla comunicazione con passeggeri del veicolo stesso.

In ogni caso tutto ciò è possibile unicamente per i progressi compiuti dall'elettronica, soprattutto nell'ultimo ventennio.

A questo proposito può essere interessante osservare che la realizzazione delle molteplici funzioni che abbiamo brevemente elencato è essenzialmente legata a $\mathrm{mn}$ particolare aspetto della evoluzione dell'elettronica: la possibilità di realizzare con sufficiente grado di aflidamento delle apparecehiature destinate a funzioni complesse.

Benchè sia difficile esprimere sinteticamente l'evoluzione dell'elettronica, tuttora in atto, essa puo forse essere indicata con un esempio 
di camattere generale: dal 1920 ad oggi il numero dei componenti di un radiotrasmettitore è aumentato, in media, secondo un fattore di circa 10 per decemnio.

Si tratta, ovviamente, di una valutazione estremamente sommaria: si può tuttavia osservare che forse essa è ancora in difetto, in quanto leelettronica is sempre più orientata verso apparecechiature a funzioni qualitativamente limitate, anche se quantitativamente più estese.

Questo sviluppo, con applicazioni che una volta sarebbero parse impossibili, dà luogo ad altri gravi problemi. Tră questi i più importanti sono, certamente, da un lato la necessità di conservare e possibilmente migliomare il gratlo di afficlamento delle apparecchiature e, dallabltro, la necessità di contenere il volume rlegli apparati ed il loro fabbisogno di alimentazione alettrica entro limiti ragionevoli, pur in presenza del numero crescente dei componenti.

Per eiò che si riferisce al volume degli apparati ed al fabbisogno di alimentazione crediamo di poter dire che le prospettive appaiono oggi farorevoli; sia per le nuove tecniche di microminiaturizzazione dei componenti; sia per le possibilita di alimentazione con energia immagazzinata o convertita da energia esterna.

Anche per il grado di afficlamento la risposta e rassicumante: nonostante il continuo aumento del numero di eomponenti delle apparecechiatture elettroniche l'incirlenza delle avarie (esperessa, per esempio, col numero probabile di ore di funzionamento tra due avarie suceessive, per un dato tipo di apparecohiature e in determinate condizioni di impiego) è rimasta finora pressochè immutata. E difficile affermare semz'altro che questa situazione potrà essere mantenuta o migliorata in futuro, con il continuo aumento della complessità degli apparati, che può essere dato per scontato. Tuttavia le teconiche specifiche dei componenti (e principalmente le tecniche dell'elettronica allo stato solido), la teconica di migliomanento delle condizioni ambientali, le tecniche di controllo funzionale rapido, in condizioni eventualmente più critiche di quelle di impiego effettivo, ed infine quelle dei circuiti di tipo fail-safe hamno certamente contributo e continueranno in larga misura a contribuire alla possibilita, per l'elettronica, di fornire il suo indispensabile contributo al progresso della tecricar aerospaziale. 